\title{
Ryegrass endophyte: host/fungus interaction
}

\section{JAN SCHMID $^{1}$ and MICHAEL J. CHRISTENSEN ${ }^{2}$}

\author{
${ }^{1}$ Institute of Molecular BioSciences, Massey University, Private Bag 11222, Palmerston North \\ ${ }^{2}$ AgResearch Grasslands, Private Bag 11008, Palmerston North \\ 1J.Schmid@massey.ac.nz
}

\begin{abstract}
This paper provides a brief introduction to the biology of endophyte-ryegrass associations and summarises results of our recent research into the interactions between host and endophyte. Specific topics include: (i) interactions between fungus and plant necessary for their successful association, (ii) the levels of biological activity of endophyte hyphae in the plant, and (iii) the potential of endophyte-ryegrass associations to protect themselves against accumulation of detrimental mutations in the endophyte and to benefit from beneficial mutations in the endophyte.
\end{abstract}

Keywords: clonal selection, compatibility, endophyte-plant interaction, endophyte metabolism, endophyte-plant signalling, evolution, mutation, Neotyphodium, reporter gene, synchronous growth

\section{Basic features of the symbionts and the symbiosis}

Endophytes are fungi and as such are actually more closely related to plants than to many other microorganisms symbiotic with plants, such as bacteria (Madigan et al. 1997a). As a result their cells share many features with plant cells - apart from some differences in chemical composition. One of the two major differences between a fungal cell and a plant cell is that the former does not contain chloroplasts. Fungi can therefore not carry out photosynthesis and, like ourselves, have to feed off carbon compounds which they acquire from their environment. The second major difference between a plant cell and a fungal cell is size; as shown in Figures 1 to 3, fungal cells are considerably smaller than plant cells. An additional difference between plants and fungi concerns the arrangement of cells; plant cells form multilayered tissues, whereas fungal cells are arranged in strands called hyphae.

Also depicted in Figure 1 is the arrangement of fungal hyphae within ryegrass leaf tissue. Unlike many plant pathogens, endophytic fungi do not invade plant cells; rather they grow in the spaces between them, where they take up nutrients that pass through these spaces. The surrounding plant cells do not show any of the alterations typical of plant defence against invading pathogens such as localised cell death (Christensen 1995), although in some novel associations host responses do occur (Christensen 1997). Figure 1 also gives some impression of the ratio between plant biomass and fungal biomass. Even in those parts of the leaf which contain the highest concentration of hyphae, the fungus only constitutes a negligible part of the biomass - less than 2\% (Ball et al. 1995; Tan et al. 1997; Schmid et al. 2000).

Figure 1 A 3-D diagrammatic close up of endophyte-infected grass tissue. The rod-shaped endophyte hyphae run, parallel with the longitudinal axis of the leaf, within the spaces between the much larger block-shaped plant cells.

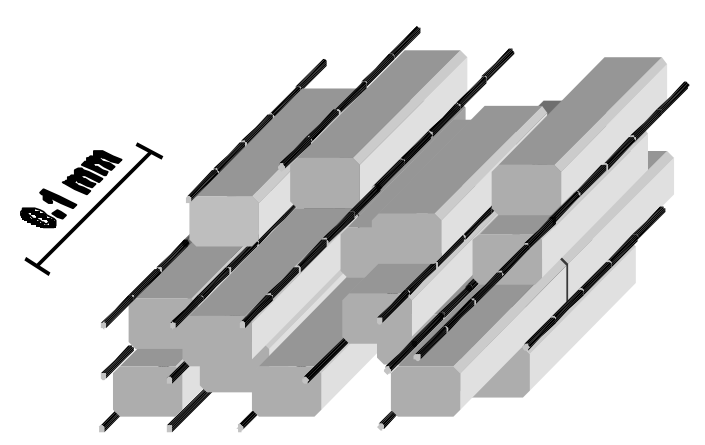

The symbiosis: endophyte distribution and growth, and endophyte-host compatibility

Much of the collaborative effort of our groups at Massey and AgResearch has focussed on determining the distribution of endophyte hyphae in ryegrass, the control of this distribution, and the factors governing the compatibility between endophyte and host (Koga et al. 1993; Christensen 1995; Christensen et al. 1997; Herd et al. 1997a,b; Tan et al. 1997; Spiering 1999; Tan et al. 1999; Schmid et al. 2000). In associations we have investigated, a characteristic pattern is observed. In vegetative tillers, the concentration of endophyte hyphae is highest in the leaf sheath ${ }^{1}$ - typically several hundred hyphae can be seen (more or less evenly

1 In reproductive tillers, the developing inflorescences may contain an even higher concentration of endophyte hyphae than the leaf sheaths. 
distributed) in a sheath cross section. In contrast, leaf blades typically contain less than 100 hyphae (predominantly in areas surrounding the nutrientcarrying vascular bundles; Figure 3), and at their tips the number is reduced to 10 or less. Remarkably, this distribution pattern is already observable in the young leaf which is still growing, and the concentration of hyphae in a leaf does not increase (or decrease) significantly after it has matured. This consistency implies that both endophyte hyphae and leaf tissues must grow synchronously, i.e., they must extend at the same rate and start and stop growth at approximately the same time ${ }^{2}$. This synchronous growth is remarkable, considering that leaves and hyphae have very different methods of extension. Whereas the leaf grows by addition and subsequent expansion of new cells at its base (Soper \& Mitchell 1956), fungal hyphae extend by adding material to their tips (Bartnicki-Garcia 1973). This implies that the entire length of the endophyte hypha must continuously slide along the adjacent plant cells while the leaf grows (Figure 4). This synchronous growth of leaf and endophyte seems to be a characteristic of all natural and all functional artificial grass-Neotyphodium associations, and also of associations involving the related Epichloe or "choke" fungi.

Figure 2 Electron microscopy image of a cross section of a ryegrass leaf blade showing three hyphae $(\rightarrow)$ against chloroplast-containing leaf cells (4500x).

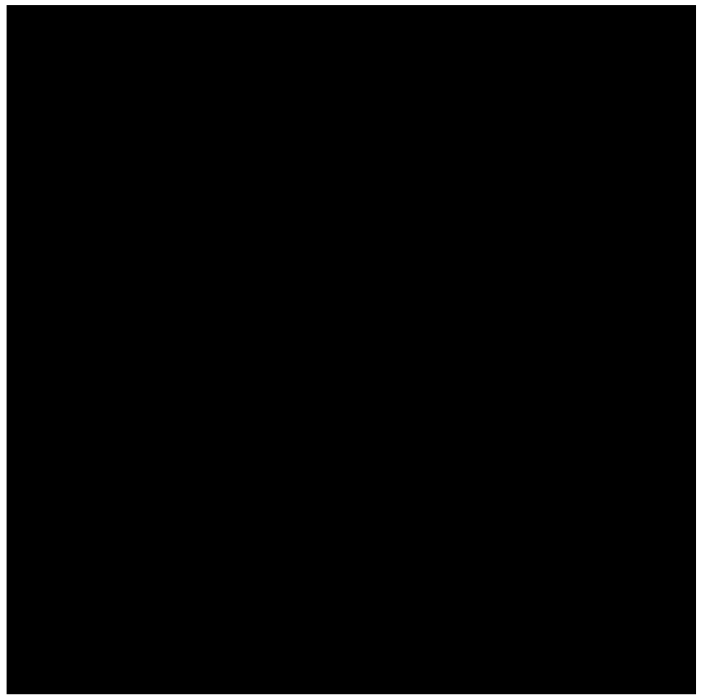

Figure 3 Light microscopy image of cross section of a ryegrass leaf blade showing endophyte hyphae $(\rightarrow)$ surrounding a vascular bundle through which plant nutrients and water are distributed around the plant (500x).

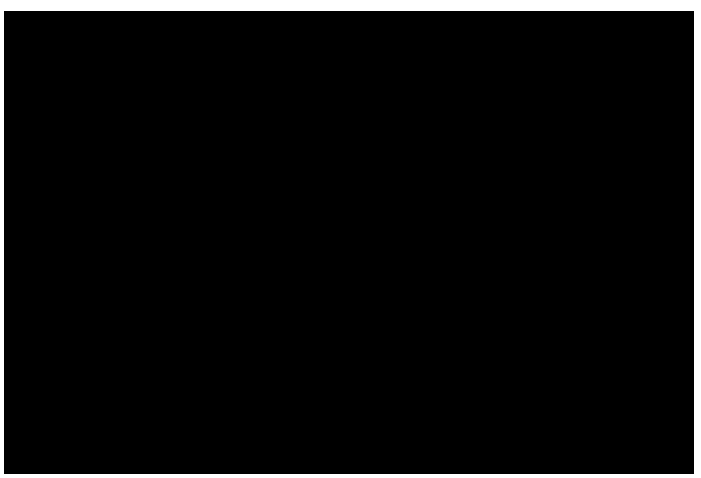

Figure 4 Assumed pattern of simultaneous growth of endophyte and plant tissue during leaf formation. As the endophyte adds new cells to the tip of its hypha and the plant adds new cells at the bottom of the leaf, the originally adjacent cells within hypha and plant (on the left, marked with white markers) become progressively separated.
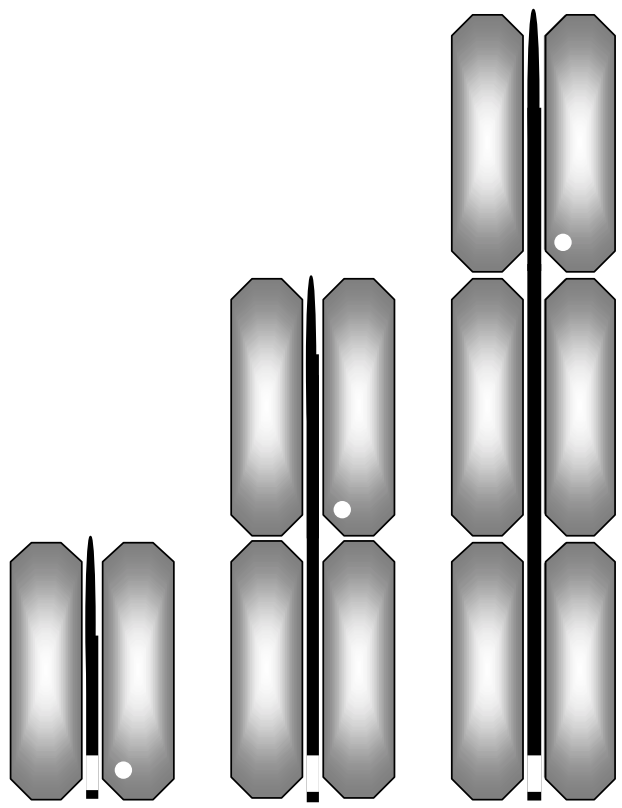

$2 \quad$ Not all endophytic fungi stop growing when leaf extension is complete. The so called P- endophytes, which are also fungi but only distantly related to the Neotyphodium endophytes, continue to proliferate after leaf extension has stopped. Continued proliferation has also been observed for a mutant strain of $N$. lolii (Christensen 1997). 
It is believed that the ancestors of the ryegrass endophytes were plant pathogenic fungi that caused damage to the plant by excessive growth. Today's endophytes have no such ill effects because the extent, timing and location of endophyte growth is tightly coordinated with the growth of the host plant. A question that interested us was how this is achieved. One possibility is that the plant controls the growth of the endophyte by regulating the amount of nutrient it supplies to the hyphae, providing nutrients where endophyte growth is required and starving the hyphae when growth is to be stopped. To test this hypothesis, we labelled endophytes with a so called 'reporter gene' (Murray et al. 1992; Herd et al. 1997a,b). Such labelled endophytes produce a fluorescent product, and the amount of fluorescence depends on their metabolic rate. To our surprise, we found no significant differences in the metabolic activity of the endophyte between different parts of the plant or between leaves of different ages. Regardless of whether or not the endophyte is actively growing in the plant, its metabolism is highly active. These observations suggest that regulatory pathways, rather than nutritional or physical constraints, are responsible for cessation of growth in normal Neotyphodium-ryegrass associations. From this we conclude that the two organisms have learned to exchange signals which guide the endophyte's growth to their mutual benefit.

We do not know the nature of the plant signals guiding endophyte growth, but it is clear that these control mechanisms are not merely a simple on/off switch for endophyte growth; they must also regulate morphological features of the endophyte hyphae. We mentioned earlier that the entire length of the hypha needs to slide through the adjacent tissue to allow the endophyte to grow synchronously with the plant. Most fungi would not be able to accomplish this because their hyphae form branches at regular intervals, which would tend to hook the hypha into place, and would result in its rupture during leaf extension. Endophytic fungi do branch freely in laboratory culture, and must do so within the apical meristem (that is the growing point at the base of the tiller, from which the leaf primordia, structures that eventually become new leaves are formed), but do not do so when growing in the leaves. Presumably they have developed regulatory mechanisms which allow them to 'switch off' branching during growth within the leaf.
Different functional associations do not have identical distributions of endophyte hyphae. Rather, while all associations follow the general pattern described above, different associations differ in the overall concentration of hyphae within their leaves as well as their distribution, with some associations having endophyte-free ${ }^{3}$ leaf blades. Again we do not know how these differences between associations arise but we do know that the genotype of both plant and endophyte play a role. The same endophyte strain can show different patterns of distribution in different plants of the same cultivar, but between tillers derived from the same plant the distribution of endophyte hyphae is always quite similar ${ }^{4}$. Likewise different endophyte strains have distinct distribution patterns in the same host species. Possibly related to the regulation of endophyte growth is a recent observation (M. Christensen unpublished data) that the host genotype also influences the percentage of seeds which, when germinating, still contain viable endophyte hyphae. One explanation for this phenomenon could be that the plant genotype determines the number of hyphae entering the seed when it is formed - the more hyphae a seed contains, the more likely it is that some hyphae will still be viable when it germinates.

Another important feature of the symbiotic relationship between plant and endophyte is the avoidance of host responses such as localised cell death, which plants use to eliminate pathogenic invaders. Research on artificial associations has shown that Neotyphodium endophytes are quite capable of eliciting such responses and their hosts capable of producing them (Koga et al. 1993; Christensen 1995). In functional associations both partners must have thus acquired ways to avoid the triggering of such responses.

A final point in considering control signals between plant and endophyte is the possibility that the endophyte might exert some control over the growth of the plant. It is known that endophytic fungi can produce compounds that function as plant growth regulators (West 1994); and effects of endophytes on plant growth have been observed (Latch et al. 1985).

The picture that emerges is that of a highly developed, complex, symbiotic interaction. Today's natural associations have presumably co-evolved from common ancestral associations and have had millions of years to perfect these interactions ${ }^{5}$. Therefore, it is almost

Or at least such a low concentration of hyphae that they are not detectable by our methods.

4 That this is a genetic effect is demonstrated by the observation that the distribution characteristics persist in lines of plants that have been vegetatively propagated over several years. This can be demonstrated when plants are maintained at constant environmental conditions. Experiments in the field and in the glasshouse show that the patterns are subject to seasonal variation.

5 This long coexistence and coevolution can be deduced from the narrow host range of Neotyphodium endophytes. Not only are these endophytes confined to temperate C3 grasses within one grass subfamily (the Pooideae), but every Neotyphodium species is only found on one particular species (Christensen et al. 1993); it is assumed that both the grass species and their endophyte species have evolved together from a common ancestor. 
surprising that our attempts to create novel associations have met with success. Presumably novel associations are possible because fundamental rules and signals exist which are common to all successful associations. Nevertheless, there are limits to our ability to produce new associations. Neotyphodium endophytes can only be successfully transferred into new hosts that are fairly closely related to their original host, and even then the association may not be functional, due to death of endophyte hyphae, death of host tissue or poor seed transmission of the endophyte.

\section{The chemical factory}

The endophyte produces within the plant a large number of chemical substances. The agriculturally-important effects of some of these substances are discussed by Lane (1999). In addition, other compounds such as antifungal substances (Christensen 1996) and plant growth regulators are made by the endophyte, and these may also affect the performance of associations (West 1994). These substances are so-called secondary metabolites, a name given to all compounds which are not essential to the growth of the microbe that produces them. It is generally assumed that the formation of such secondary metabolites mainly occurs in response to starvation - and this is supported by a large body of experimental evidence collected on laboratory cultures of a variety of microbes (Madigan et al. 1997).

Considering that even a large ryegrass tiller contains only a few milligrams of endophyte, endophytes often produce copious amounts of secondary metabolites. Since starvation would tend to slow down the metabolism of the endophyte, the large amounts of secondary metabolites produced by the endophyte in the plant seemed difficult to explain. Our work on the metabolism of endophytes has provided an unexpected ${ }^{6}$ and very simple explanation for the timing and the amount of the production of these compounds. As explained above, endophyte hyphae extend synchronously with the growing leaf tissue. This places considerable demands on their metabolism, which needs to produce new cells at the same rate as the surrounding plant cells, leaving little room for the diversion of the endophyte's metabolism towards production of chemical agents. However, as explained earlier, after cessation of fungal growth when the leaf is mature, the metabolic rate of the hypha does not slow down. A large part of the endophyte's metabolic energy ${ }^{7}$ can then be directed towards the production of chemical compounds aimed at protecting the plant from herbivores and insect pests. Thus, the same controls that synchronise endophyte and plant growth to prevent plant damage due to excessive hyphal proliferation, and may also ensure that the plant is able to fully exploit the endophyte as a 'biochemical factory' for its protection.

There are likely to be additional controls influencing the amount and the number of compounds produced. Different associations have different profiles of toxins and pest deterrents (Christensen et al. 1993) and even the same endophyte strain can produce a different mix of these compounds in different grass genotypes (Spiering 1999). Likewise environmental parameters (such as fertilisation and temperature) apparently influence the synthesis of these compounds. At present we can only measure their distribution within the plant, which may not necessarily correspond to the site of synthesis. Part of our future work, using reporter gene technology, is aimed at measuring to what degree regulation of endophyte genes through plant and endophyte signals is responsible for these effects.

\section{Maintenance and improvement of the fitness of associations by selection}

All organisms are subject to spontaneous mutations. Fungi like the ryegrass endophytes can be expected to acquire between 0.0002 and 2 mutations per cell division (Madigan et al. 1997b), that is, every time a hypha adds a new cell to its tip. By the time the total length of a network of endophyte hyphae has reached $10 \mathrm{~cm}$, it may have incurred at least one mutation and may (theoretically) have accumulated as many as 10000 , since roughly 50 cells have to be added for every $\mathrm{mm}$ of growth. To put these numbers into perspective, consider that a single leaf sheath contains several hundred hyphae that may possess a combined length of more than a meter.

This is not quite as dramatic as it sounds. Firstly, even 10000 mutations only represent an alteration of less than $0.1 \%$ of the endophyte's genetic information. Secondly, many mutations have no effect on the functionality of the endophyte's cellular machinery ${ }^{8}$. In addition, deleterious mutations which severely interfere

\footnotetext{
6 Cessation of growth of a fungal hypha without slowing down its metabolism has, to our knowledge, never been described before. Usually hyphae will stop growing only if their metabolism slows down.

7 Some "maintenance biosynthesis" of items such as enzyme molecules is still required, in addition to secondary metabolite production.

8 Either because they occur in regions of the endophyte's DNA which do not encode products (so called 'junk DNA') or because they do not change the product made from the gene (genetic information is encoded in base triplets, but the code is redundant so that in some cases two different triplets contain the same information. A mutation changing one triplet into another that contains the same information will not affect the product made by the gene).
} 
with the ability of an affected hypha to proliferate in the plant, will likely lead to the elimination of that hypha and its mutation.

However, mutations that do not negatively affect hyphal proliferation will persist within the tiller. An obvious example would be mutations that affect the synthesis of herbivore deterrents by the association. It would benefit associations if mutations that enhance their survival are maintained. It is also crucial that mutations that negatively affect their survival are not allowed to accumulate. Using our artificially introduced reporter gene as a model, we have shown that associations employ a method of clonal selection to accomplish this. Apparently new vegetative tillers are colonised by hyphae originating from a single hyphal nucleus in the maternal tiller so that all of them will contain the mutations present in that parental nucleus. If these hyphae in the new tiller contain a mutation which will enhance the fitness of the tiller over that of other tillers, the tiller (and its future progeny) will have a selective advantage over other tillers, and the 'improved' association will outperform the original and replace it over time. If the mutation was detrimental (and most mutations are) the progeny of the affected tiller will be eliminated by competition with tillers of the unaltered associations. These conclusions are based on both theoretical considerations and the behaviour of the artificially introduced reporter gene. This gene places a small metabolic burden on the endophyte and tends to be eliminated during vegetative propagation of plants. We can deduce that clonal selection occurs during vegetative propagation of tillers because the progeny of plants infected with an endophyte carrying the reporter gene produce new tillers in which either all or none of the endophyte hyphae contain the reporter gene. Tillers containing a mixture of hyphae with and without the reporter gene seem to be extremely rare and have so far not been observed in our experiments. We have not yet investigated whether the clonal selection process also occurs as part of endophyte seed transmission but have reason to believe that it might.

We believe that through this selection process ryegrass endophyte associations have improved their fitness over millions of years and maintain it today. An important implication of this mechanism is that the endophytes in both natural and novel associations are presumably not genetically uniform but consist of an array of competing lineages ${ }^{9}$. The existence of such gene pools offers the potential to further improve existing and novel associations through continuing selection. On the other hand, since not all mutations that enhance the survival of an association in the field are agricul-turally desirable, continued monitoring and selection may be important for maintaining the full benefit of associations in the field. Currently monitoring and selection can only be carried out by assessing the actual performance of associations. However, if these evaluations are combined with new high-resolution DNA typing techniques (Vos et al. 1995) currently being adapted for use with endophytes by AgResearch, we can hope to discover molecular markers that allow us to predict the performance of endophyte lineages and to select the best lineages more efficiently ${ }^{10}$. Note that knowing what genes are responsible is not a prerequisite for the selection process. All that is required is to obtain a random selection of genetic markers and to test whether the presence of some of them correlates with desirable properties. Such correlations occur if a marker is located in the vicinity of a gene that affects the performance of the association. Markers can therefore also be used to identify these genes at a later stage.

\section{ACKNOWLEDGEMENT}

We thank the Foundation for Research Science and Technology for supporting this research.

\section{REFERENCES}

Ball, O.J.-P.; Prestidge, R.A.; Sprosen, J.M. 1995. Interrelationships between Acremonium lolii, peramine and lolitrem $\mathrm{B}$ in perennial ryegrass. Applied and Environmental Microbiology 61: 15271533.

Bartnicki-Garcia, S. 1973. Fundamental aspects of hyphal morphogenesis. pp. 245-267 In: Microbial Differentiation. $23^{\text {rd }}$ Symposium of the Society for General Microbiology. Imperial College. University Press, London.

Christensen, M.J. 1995. Variation in the ability of Acremonium endophytes of Lolium perenne, Festuca arundinacea and $F$. pratensis to form compatible associations in the three grasses. Mycological Research 99: 466-470.

Christensen, M.J. 1996. Antifungal activity in grasses infected with Acremonium and Epichloë endophytes. Australasian Plant Pathology 25: 186-191.

$9 \quad$ Theoretical considerations suggest that in long established associations a limited number of optimal endophyte genotypes (evolved over long periods of coevolution) will probably continually predominate, unless new selection pressures arise. In novel associations, in which the partners are not as fine-tuned to each other, random mutations would be more likely to generate lineages which can outperform and thereby replace the original association.

10 This approach has been very successful in the New Zealand forestry industry. 
Christensen, M.J. 1997. Endophyte compatibility in perennial ryegrass, meadow fescue and tall fescue, a short review. pp. 45-48 In: Neotyphodium/Grass Interactions. Eds. Bacon, C.W.; Hill, N.S. Plenum Press, New York \& London.

Christensen, M.J.; Ball, O.J.-P.; Bennett, R.J.; Schardl, C.L. 1997. Fungal and host genotype effects on compatibility and vascular colonization by Epichloe festucae. Mycological Research 101: 493-501.

Christensen, M.J.; Leuchtmann, A.; Rowan, D.D.; Tapper, B.A. 1993. Taxonomy of Acremonium endophytes of tall fescue (Festuca arundinacea), meadow fescue (F. pratensis), and perennial ryegrass (Lolium perenne). Mycological Research 97: 1083-1092.

Herd, S.; Christensen, M.J.; Saunders, K.; Scott, D.B.; Schmid, J. 1997a. Quantitative assessment of in planta distribution of metabolic activity and gene expression of an endophytic fungus. Microbiology 143: 267-275.

Herd, S.; Christensen, M.J.; Saunders, K.; Scott, D.B.; Schmid, J. 1997b. Quantitative assessment of in planta distribution of metabolic activity and gene expression of Neotyphodium endophytes. pp. 57-60. In: Neotyphodium/Grass Interactions. Eds. Bacon, C.W.; Hill, N.S. Plenum Press, New York \& London.

Koga, H.; Christensen, M.J.; Bennett, R.J. 1993. Incompatibility of some grass-Acremonium endophyte associations. Mycological Research 97: 1237 1244.

Lane, G.A. 1999. Chemistry of endophytes: patterns and diversity. Ryegrass endophyte: an essential New Zealand symbiosis. Grassland Research and Practice Series No. 7: 85-94.

Latch, G.C.M.; Hunt, W.F.; Musgrave, D.R. 1985. Endophyte fungi affect growth of perennial ryegrass. New Zealand Journal of Agricultural Research 28: 165-168.

Madigan, M.T.; Martinko, J.M.; Parker, J. 1997a. p. 623. In: Biology of Microorganisms, $8^{\text {th }}$ edn. Prentice-Hall International, Inc., Englewood Cliffs, New Jersey.

Madigan, M.T.; Martinko, J.M.; Parker, J. 1997b. p. 309. In: Biology of Microorganisms, $8^{\text {th }}$ edn.
Prentice-Hall International, Inc., Englewood Cliffs, New Jersey.

Murray, F.R.; Latch, G.C.M.; Scott, D.B. 1992. Surrogate transformation of perennial ryegrass, Lolium perenne, using genetically modified Acremonium endophyte. Molecular and General Genetics 233: 1-9.

Schmid, J.; Spiering, M.J.; Christensen, M.J. 2000. Metabolic activity, distribution and propagation of grass endophytes in planta. Investigations using the GUS system. pp. 295-322. In: Microbial Endophytes. Eds. White, J.F.; Bacon. C.W. Marcel Dekker, New York.

Soper, K.; Mitchell, K.J. 1956. The developmental anatomy of perennial ryegrass (Lolium perenne L.). New Zealand Journal of Science and Technology 37: 484-504.

Spiering, M. 1999. Distribution of Neotyphodium endophyte metabolic activity in ryegrass and its impact on alkaloid distribution and photosynthesis. $\mathrm{PhD}$ thesis. Massey University.

Tan, Y.Y.; Spiering, M.; Christensen, M.J.; Saunders, K.; Schmid, J. 1997. In planta metabolic state of Neotyphodium endophyte mycelium assessed through use of the GUS reporter gene in combination with hyphal enumeration. pp. 85-87. In: Neotyphodium/Grass Interactions. Eds. Bacon, C.W.; Hill, N.S. Plenum Press, New York \& London.

Tan, Y.Y.; Spiering, M.; Lane, G.; Christensen, M.J.; Schmid, J. 1999. Metabolic state of Neotyphodium endophyte mycelium in planta assessed using the GUS reporter gene system and hyphal enumeration. In preparation.

Vos, P.; Hogers, R.; Bleeker, M.; Reijans, M.; van de Lee, T.; Hornes, M.; Frijters, A.; Pot, J.; Peleman, J.; Kuiper, M. 1995. AFLP: a new technique for DNA fingerprinting. Nucleic Acids Research 23: 4407-14.

West, C.P. 1994. Physiology and drought tolerance of endophyte-infected grasses. pp. 87-99. In: Biotechnology of endophytic fungi of grasses. Eds. Bacon, C.W.; White J.F. Jr. CRC Press, London. 